\title{
The use of eHealth and digital technologies in the Italian geriatric practice: a survey of the Italian Geriatric Society (SIGOT)
}

\begin{abstract}
Alberto Cella, ${ }^{1}$ Erica Volta, ${ }^{1}$
Jacopo Demurtas, ${ }^{2,3}$ Renato Zaccaria, ${ }^{4}$

Moira Ceci, ${ }^{5}$ Andrea Pilotto, ${ }^{6}$

Virginia Boccardi, ${ }^{7}$ Alberto Castagna, ${ }^{8}$

Carlo Custodero, ${ }^{9}$ Francesco Vetta, ${ }^{10}$ had increased significantly due to COVID-19 pandemic and for $80 \%$ of the members, the priority areas for the use of technologies for the care and treatment of the elderly are the management of chronic diseases and the prevention of hospitalization.
\end{abstract}

Marina Barbagelata, ${ }^{1}$ Nicola Veronese, ${ }^{11}$

Alberto Pilotto ${ }^{1,9}$

${ }^{1}$ Department Geriatric Care,

Orthogeriatrics and Rehabilitation, E.O.

Ospedali Galliera, Genova; ${ }^{2}$ Clinical and

Experimental Medicine PhD Program,

University of Modena and Reggio

Emilia, Modena; ${ }^{3}$ Primary Care

Department, USL Toscana Sud Est-

Grosseto, Grosseto; ${ }^{4}$ Department of

Informatics, Bioengineering, Robotics, and Systems engineering (DIBRIS), University of Genoa, Genoa; ${ }^{5}$ Local Health Agency ULSS 18, Rovigo; ${ }^{6}$ Neurology Unit, Department of Clinical and Experimental Sciences, University of Brescia, Brescia; ${ }^{7}$ Department of Medicine, Institute of Gerontology and Geriatrics, University of Perugia, Perugia; ${ }^{8}$ Center for Cognitive Disorders and Dementia, ASP Catanzaro,

Catanzaro; ${ }^{9}$ Department of

Interdisciplinary Medicine, University of

Bari Aldo Moro, Bari; ${ }^{10}$ Saint Camillus

International University of Health

Sciences, Rome; ${ }^{11}$ Geriatric Unit,

Department of Internal Medicine and

Geriatrics, University of Palermo,

Palermo, Italy

\section{Abstract}

e-Health technologies may potentially improve healthcare assistance for frail elderly subjects. However, despite the interest and potential benefits of the field, research highlighted that several challenges remain, such as scarce wide spreading of information and communication technology technologies among the elderly and infrastructural difficulties.

The aim of this survey was to assess to what extent digital technologies are widespread and used among Italian geriatricians.

We conducted an online survey addressed to geriatricians, from the SIGOT (The Italian Geriatric Society) website, collecting the 78 completed questionnaires. We then analyzed with descriptive statistics the data.

Results showed a still scarce use of digital technologies in geriatric practice in Italy. However, more than $60 \%$ of the SIGOT geriatricians who participated in the survey responded that the use of digital technology

\section{Introduction}

Considering the rising of older population, ${ }^{1,2}$ one of the most important challenge societies all around the world must face is the increasing prevalence of frailty among elderly people. ${ }^{3}$ Frailty is a multidimensional syninclude dependency, vulnerability, chronic disease, reduced quality of life, long-term hospitalization and death. ${ }^{4}$ Very often it happens that frail people have more than one chronic condition, with the consequence of requiring continuous care and monitoring of their health status. ${ }^{5}$ Furthermore, the rapid growing of older population means an increasing demand of care and health services for elderly.

Concurrently, in last years, the development of information and communication technology (ICT) represented an important opportunity to provide new ways to improve the quality of life for the elderly and monitor their health status. ${ }^{6}$ Against the backdrop of limited capacity and weakening of familybased care systems, developing e-health technologies has emerged as a possible solution. Nowadays, telecare, telemedicine and telehealth are concepts that have entered the everyday information system and research has long been exploring such solutions. These innovative methods are often used. $^{7}$ Technologies comprised under this broad umbrella range from extending the in-person visits using solutions such as videoconferencing to solutions such as wearable technologies. Those solutions require minimal interaction with the patient to capture, store, and transmit vital patient-generated data. More sophisticated solutions, such as so-called smart homes, are also being actively developed and explored. ${ }^{8}$ Digital health technologies have the potential to assist oncologists in more intensive and robust monitoring of older patients with cancer. However, there are still many challenges in implementing these solutions as a part of routine medical care, particularly for specific populations such as drome occurring in the elderly; its symptoms
Correspondence: Alberto Cella, Department Geriatric Care, Orthogeriatrics and Rehabilitation, E.O. Ospedali Galliera,

Genova, Italy.

Tel.: +39.010.5634871.

Fax: +39.010 .57481020 .

E-mail: alberto.cella@galliera.it

Key words: Elderly; gerontology; technology; tele-medicine; tele-assistance; survey.

Conflict of interest: the authors declare no potential conflict of interest.

Funding: this survey received an unconditional grant from Teseo S.r.l., Genoa, Italy.

Received for publication: 25 November 2021. Accepted for publication: 3 December 2021.

This work is licensed under a Creative Commons Attribution-NonCommercial 4.0 International License (CC BY-NC 4.0).

${ }^{\circ}$ Copyright: the Author(s), 2021

Licensee PAGEPress, Italy

Geriatric Care 2021; 7:10301

doi:10.4081/gc.2021.10301

older adults, who may have a lower previous exposure to technology. Telemonitoring is one of the essential telehealth methods, that has widespread applications in health monitoring application field. ${ }^{9}$ Furthermore, due to COVID-19 pandemics, a lot of researches emerge exploiting telemonitoring as effective way to reduce hospitalization for COVID-19 patients. ${ }^{10,11}$ Literature shows that telemonitoring is an essential element to providing satisfactory care to elderly people. It permits, in fact, frail elderly people to live independently in their own home, but with a direct contact to specialists, relatives and caregivers. ${ }^{12}$ Furthermore, telemonitoring would be also a good option for addressing the increasing costs associated to the healthcare of frail elderly patients..$^{13}$ This type of solution, indeed, let to decrease the number of visits and costs, improve the overall quality of life and allow patients to stay in their residency. 5,14

e-Health solutions like telemonitoring, however, requires suitable systems to provide and manage the needed care for age-related chronic diseases. In the survey we presented in this paper, we will highlight the criticism and difficulties of wide-spreading e-Health in Italian Geriatric everyday practice, due also to infrastructural fragilities. In addition, there are other challenges related to health telemonitoring systems from the patient perspectives, including a low level of education, physical weakness and multiple chronic diseases of frail people. ${ }^{15,16}$ Considering the above, it is essential a deepened analysis of 
the designing and developing interactions of these technologies, to be able to assess whether technologies meet the various requirements and needs of the different endusers (e.g., healthcare professionals, patients and caregivers). ${ }^{17-19}$ The first and core element to consider, indeed, is the promotion of Internet technologies among elderly population. A Spanish study highlighted older adults were interested in learning and acquiring ITC skills. ${ }^{20}$ In another study, ${ }^{21}$ authors reported benefits elderly with Internet access have, such as a decreasing of the risk of hypertension, ${ }^{22}$ a reducing of social isolation and depression, ${ }^{23}$ better life satisfaction, and psychological well-being among the elderly. ${ }^{24}$

A recent review ${ }^{25}$ studied in terms of effectiveness, feasibility and usability, eHealth solutions applied to geriatric rehabilitation. The survey, that included 40 studies, highlighted that at least e-Health rehabilitation is effective as traditional ones, and it seems to be feasible in geriatric rehabilitation, since none of the selected studies reported serious adverse events. For what it concerns the usability, issues were found, specifically age-related barriers. Most of all, the survey highlighted a lack of research in the field, and the importance of make healthcare professionals aware of the usability and the possibilities provided by e-Health interventions.

For the best of authors' knowledge, less research has been done in survey how physicians use e-Health and technology in everyday practice, especially in Italy. The purpose of this paper is then to explore the deployment of digital technologies among Italian geriatricians, who took part in a survey proposed by SIGOT.

\section{Materials and Methods}

During the Webinar 'Technologies and the elderly: from words to actions' organized by The Italian Geriatric Society (SIGOT), on April $22^{\text {nd }}, 2021$, a survey on the use of technologies was conducted. The audit investigated the use of technologies in the elderly, and it was conceived to capture a 'snapshot' of the real application of technology in geriatric medicine practice, according to the experience and point of view of the geriatricians of the SIGOT society.

\section{Results}

78 SIGOT members took part in the survey, with a modest prevalence of men $(56.4 \%$ of the sample).

Regarding the age groups, the most rep- resented were those between 50 and 60 years old, while the least represented (with about $6 \%$ ) were young members, under 30 , and senior members, over 70 years old.

Decades 30-40, 40-50 and 60-70 years of age are similarly represented (around 20\%). In Figure 1 a graph summarized the age groups of the survey participants.

Regarding regional origin, the geriatric physicians, that participated to our survey, reside in 17 different Italian regions and Autonomous Provinces of Italy.

Concerning the topic, the first question was related to the regular use of different digital applications, which have been potentially available to health care professionals for years., e.g., data analysis software, software for pharmacological prescriptions and digital patients' records. Results suggested that many of the members used computerized medical records, but they are still far from widespread use $(57.3 \%)$. The frequency of use of computerized tools to support clinical decisions is comparable $(54.3 \%)$, while the number of members who make use of computerized dedicated applications to prescribe therapies is significantly lower $(36.7 \%)$ (Figure 1).

Only one member over three habitually uses IT tools for the collection and analysis of clinical data (i.e., for activity reporting, budget process, clinical research, etc).

\section{RESPONDENTS BY AGE GROUP (\%)}

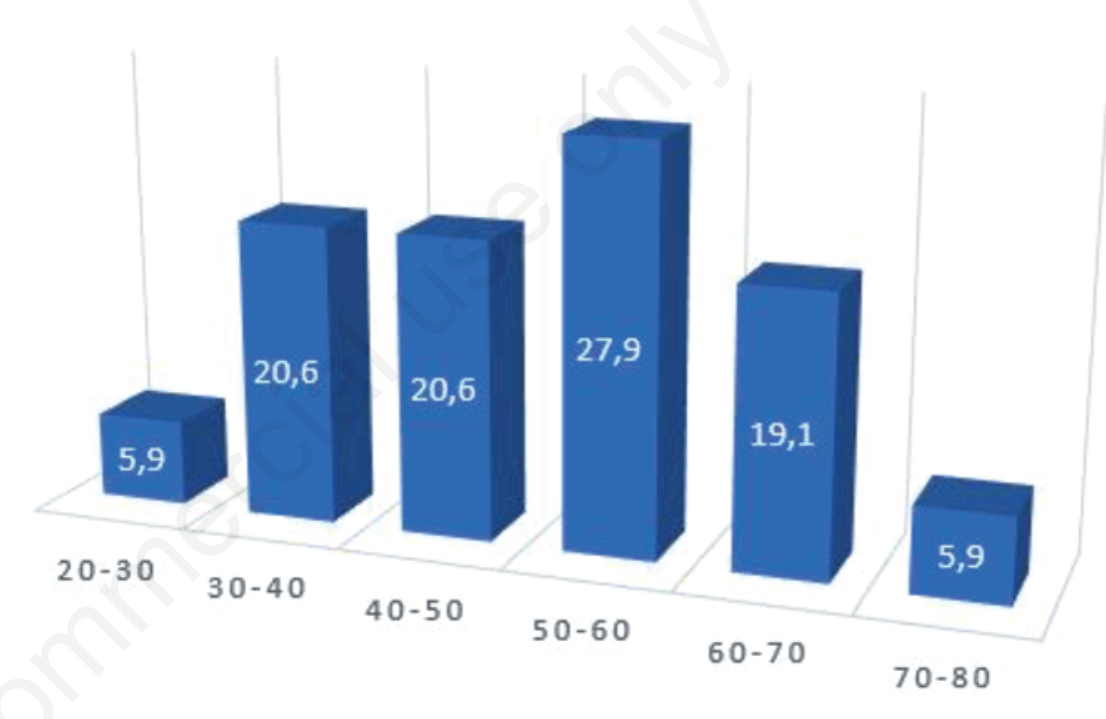

Figure 1. Age groups representation of the SIGOT members who participated to the survey, by age group.

\section{APPs AND SOFTWARES IN RESPONDENTS' CLINICAL ROUTINE}

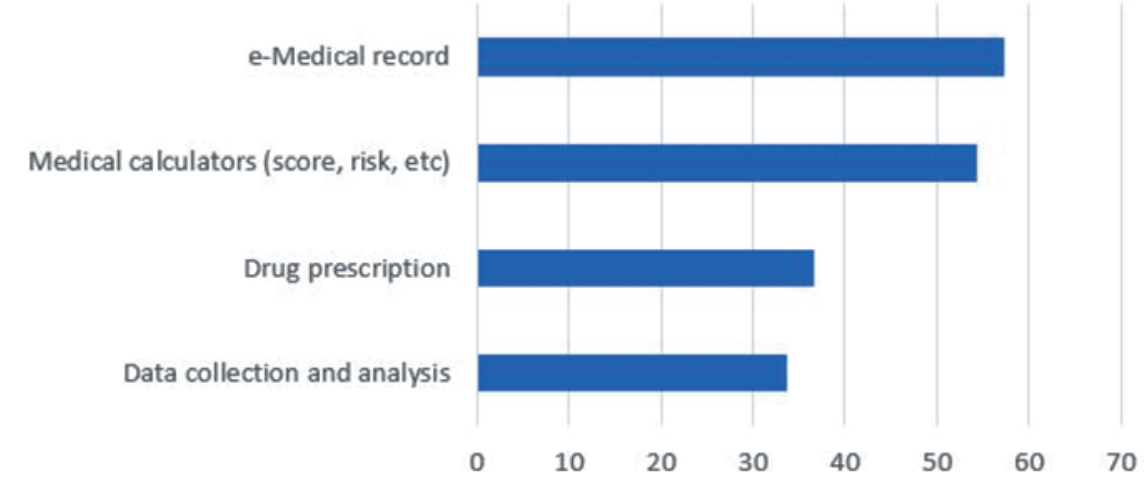

Figure 2. Apps and software used in everyday clinical routine by the SIGOT members who took the survey. 
Considering how frequent and widespread the use of video calls has been during the of the COVID-19 pandemic, firstly we asked the geriatrician who took part to the survey what may be the actual diffusion of teleconsultation and tele-visit in their professional experience: more than $60 \%$ of interviewed geriatricians declared that they do not use video-calling systems, while only $16 \%$ made use of both options; around $12 \%$ of those, regularly make video-calls and just over 10\% use teleconsultation (Figure 2).

It is not surprising, therefore, that telemedicine is already operational in only a minority of the specialist practices in which geriatricians work (about one over three), even though another $15 \%$ stated that telemedicine is expected to be activated in their own operating context in the short to medium term; conversely, in at least one case out of two of those interviewed, telemedicine does not exist and is not planned (Figure 3). If, however, we consider in a broader sense the professional relationships of geriatricians with other services of the socio-health network, the telemedicine/telecare experiences reach more than $50 \%$ (mainly as ordinary activity, but also as research activity). As expected, the most advanced technologies (assistive/rehabilitation robotics, tele-rehabilitation, domotics/environment assisted living) are used in a decidedly minority share (between 12 and $16 \%$ of respondents) and mainly for research purposes (Figure 4).

Deepening the analysis to progressively more complex and faceted aspects of the use of technologies in professional activity,

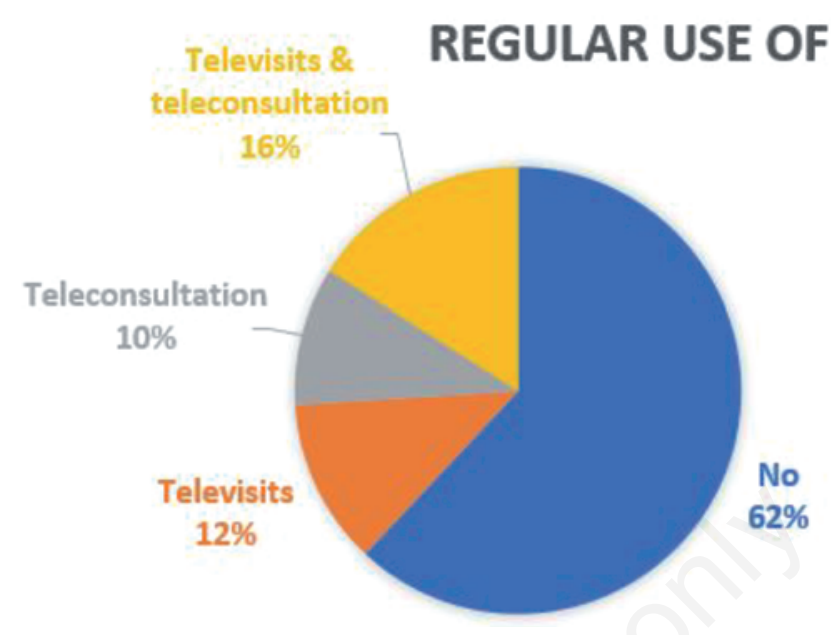

Figure 3. The graph shows how televisits and consultation (and both services) are widespread among geriatricians, due to COVID-19 pandemic.

\section{TELEMEDICINE AVAILABILITY IN THE LOCAL HEALTH SERVICE NETWORK}

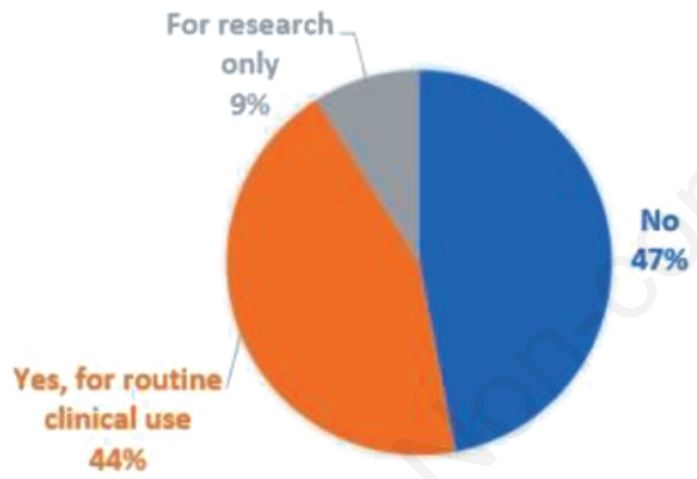

\section{TELEMEDICINE AVAILABILITY IN THE RESPONDENT'S HEALTH SERVICE}

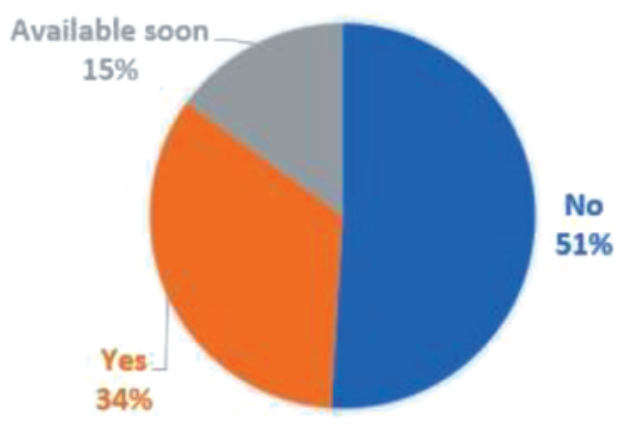

Figure 4. The chart shows the prevalence of telemedicine in geriatric medical practice and its deployment schedule across the country.

TELEREHABILITATION

- Usual clinical activity $=$ No $=$ For reaserch only

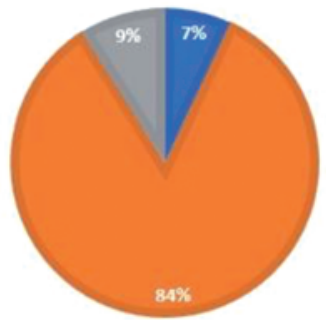

DOMOTICS/AAL

mUsual clinical activity $=$ No $=$ For reaserch only

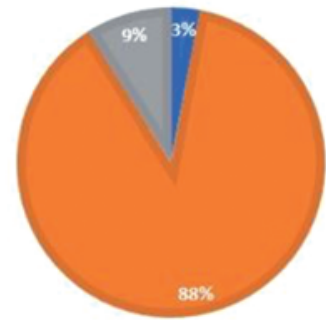

ASSISTIVE ROBOTICS/REHAB

- Usual clinical activity $=$ No $=$ For reaserch only

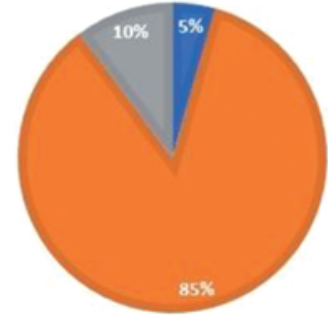

Figure 5. Results of the survey shows that the most advanced technologies (assistive/rehabilitation robotics, tele-rehabilitation, domotics/environment assisted living) are used in a decidedly minority and mainly for research purposes. 
remote monitoring of biometric parameters (blood pressure, heart rate, $\mathrm{O}_{2}$ saturation, etc.) using apps or dedicated software is practised by $30 \%$ of members (mainly in 'real-time' mode). Systems for recording and/or analyzing data collected by wearable devices (movement sensors, activity tracking, sleep, falls, cardiac arrhythmias, etc.) seem to be the prerogative of very few geriatricians in the context of ordinary activity $(4.4 \%)$ and are also poorly represented in research $(12 \%)$, as you can see in Figure 5.

We therefore wished to verify the impact of the COVID-19 pandemic on the use of technology in the management of the frail elderly: more than $60 \%$ of the SIGOT geriatricians who participated in the survey responded that it had increased significantly. And if the current use of telemedicine and eHealth in the care of the frail elderly patient is at least sufficient for only a quarter of the participants, in the organizational vision of 4 out of 5 geriatricians a high potential value of the use of telemedicine and e-Health in geriatric clinical practice is recognized.

For more than $80 \%$ of the members, the priority areas for the use of technologies for the care and treatment of the elderly are the management of chronic diseases and the prevention of hospitalization, without neglecting 'safety' in the living environment and the support of functional and cognitive deficits alongside sensory deficits (Figure 6).

Furthermore, the main actions to be implemented for a widespread diffusion of 'digital health' in geriatric care are, in the opinion of SIGOT geriatricians, the following: i) a better integration of the hospital/territory network, a prerequisite that only partly depends on technology, but which would require authoritative and unified governance at territorial level; ii) the availability of 'friendly', reliable and affordable digital devices (it should not be forgotten that the COVID pandemic has led to a substantial increase in the proportion of citizens below the poverty line); iii) the digital literacy of elderly population; iv) a better network connectivity.

Finally, contrary to what one might think,

Hospital and community health-services integration

Digital literacy (seniors)

Internet connectivity

IT standards considering the great impact of current privacy policy at national and international level, the cybersecurity aspects of digital technolo- gy seem to be of concern to only a minority of the geriatricians surveyed (about 10\%) (Figures 7 and 8).

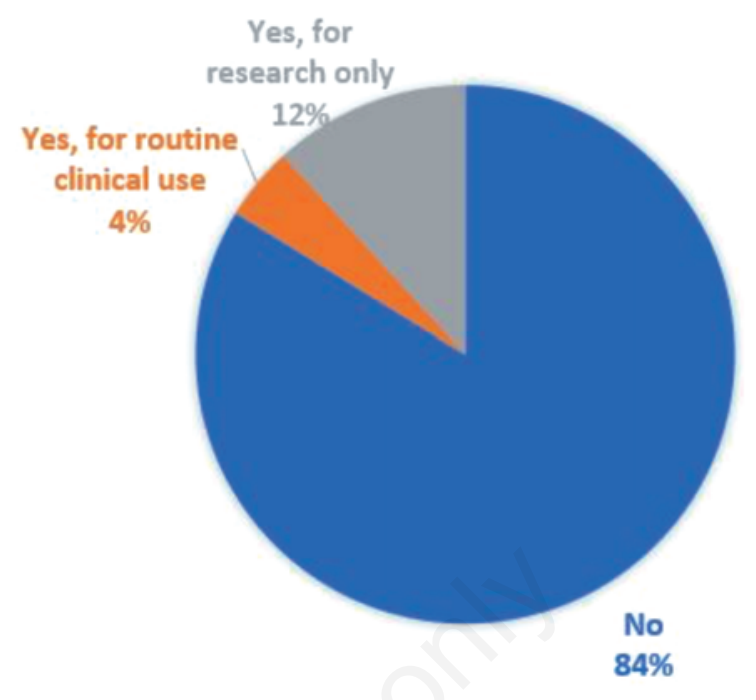

Figure 6. Representation of the diffusion in geriatric clinical practice of wearable technologies for remote patient monitoring.

\section{PRIORITIES IN THE USE OF TECHNOLOGIES IN GERIATRICS}

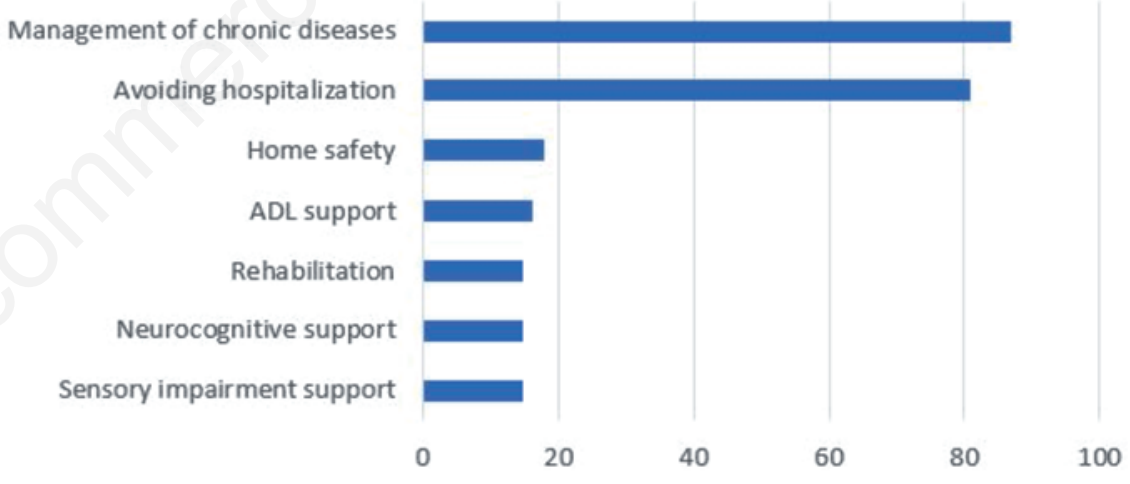

Figure 7. The SIGOT geriatricians highlighted as the priorities, in their vision, of the wide-spread of technologies in medical care. The priority areas are the management of chronic diseases the prevention of hospitalisation.

Figure 8. The main actions to be implemented for a widespread diffusion of eHealth technologies in Italian geriatric practice, in the opinion of the SIGOT geriatricians. 


\section{Discussion}

The SIGOT survey on the use of technology for the elderly is the first Italian survey to give us a snapshot of the real world in which Italian geriatric specialists work. The answers provided by them define a globally unsatisfactory picture as regards the current degree of diffusion of technologies for the elderly.

Even though there has been some impulse linked to the COVID-19 pandemic, in reality tele-visits and tele-consultations are used by less than $40 \%$ of the respondents and only in a third of the cases is an operational service available to the patients. This proportion is comparable to the one of services based on remote biometric data collection (e.g., blood pressure, heart rate, $\mathrm{O}_{2}$ saturation). The use of wearable devices for the recording of more complex clinical/functional parameters (i.e., arrhythmia, sleep quality, movements quantity and quality, falls, etc.) appears instead to be a more marginal reality, reaching about $15 \%$ of cases, including research experiences. The most advanced forms of technology (assistive/rehabilitative robotics, telerehabilitation, home automation/ambient assisted living) are also represented in a similar proportion.

Nevertheless, there is a strong positive tension in part of the SIGOT geriatricians towards the digital transformation of health services dedicated to the elderly, especially for the management of chronic diseases and the prevention of hospitalizations linked to hospitalizations linked to their relapse.

The realization of all this seems to depend not only on aspects more directly relevant to technologies (e.g., usability, purchase cost, quality of the network), but also from a more complete and functional integration of the territorial services with hospital services.

\section{Conclusions}

In the present work, we publish the results of a survey realized by the SIGOT, the Italian Geriatrician Society, to try to map and understand the actual use and deployment of eHealth and digital technologies among everyday clinical practices.

COVID-19 pandemic seemed to increase the widespread use of technology in several domain of our everyday life, but, as the survey highlighted, much work still needs to be done to truly count digital technologies (and even the more complex ones such as telerehabilitation, robotic and domotic technologies) as a widespread tool used throughout the country in geriatric practice.
As SIGOT geriatricians have pointed out, part of the difficulties to be overcome are infrastructural and nationwide, as well as related to information technology literacy of healthcare professionals that cannot be taken for granted.

To the best of our knowledge, this is a first attempt to concretely map the diffusion of technology in geriatric practice on the Italian territory. The authors sincerely hope that this initial snapshot of the reality of the clinic in the country will inspire further research work that further explores this topic.

\section{References}

1. Arif MJ, El Emary IM, Koutsouris D-D. A review on the technologies and services used in the self-management of health and independent living of elderly. Technol Health Care 2014;22:677-87.

2. Hamdi O, Chalouf MA, Ouattara D. eHealth: Survey on research projects, comparative study of telemonitoring architectures and main issues. J Network Comput Appl 2014;46:100-12.

3. Andreasen J, Lund H, Aadahl M. Content validation of the Tilburg Frailty Indicator from the perspective of frail elderly. A qualitative explorative study. Arch Gerontol Geriatr 2015;61:392-9.

4. Peters LL, Boter H, Buskens E. Measurement properties of the Groningen Frailty Indicator in homedwelling and institutionalized elderly people. J Am Med Direct Assoc 2012;13:546-51.

5. Urban M. 'This really takes it out of you!' The senses and emotions in digital health practices of the elderly. Digit Health 2017;3:2055207617701778.

6. Stowe S, Harding S. Telecare, telehealth and telemedicine. Eur Geriatr Med 2010;1:193-7.

7. Barlow J, Singh D, Bayer S. A systematic review of the benefits of home telecare for frail elderly people and those with long-term conditions. J Telemed Telecare 2007;13:172-9.

8. Santos A, Macedo J, Costa A, et al. Internet of things and smart objects for M-health monitoring and control. Procedia Technol 2014;16:1351-60.

9. Coyte PC, McKeever P. Home care in Canada: passing the buck. Can J Nurs Res Arch 2016;33:11-25.

10. Silven AV, Petrus AH, VillalobosQuesada M, et al. Telemonitoring for patients with COVID-19: recommendations for design and implementation. $\mathrm{J}$ Med Internet Res 2020;22:e20953.

11. Grutters LA, Majoor KI, Mattern ESK, et al. Home telemonitoring makes early hospital discharge of COVID-19 patients possible. J Am Med Inf Assoc JAMIA 2020;27:1825.

12. Cronfalk BS, Fjell A, Carstens N. Health team for the elderly: a feasibility study for preventive home visits. Prim Health Care Res Dev 2017;18:242-52.

13. Bobillier Chaumon M-E, Michel C, Tarpin Bernard F. Can ICT improve the quality of life of elderly adults living in residential home care units? From actual impacts to hidden artefacts. Behav Inform Technol 2014;33:574-90.

14. Lupton D. Towards sensory studies of digital health. London: SAGE Publications Sage UK, 2017.

15. Pantelopoulos A, Bourbakis NG. A survey on wearable sensor-based systems for health monitoring and prognosis. IEEE Trans Syst Man Cybern C Appl Rev 2010;40:1-12.

16. Alemdar H, Ersoy C. Wireless sensor networks for healthcare: A survey. Comp Networks 2010;54:2688-710.

17. Obrist S, Rogan S, Hilfiker R. Development and evaluation of an online fall-risk questionnaire for nonfrail community-dwelling elderly persons: a pilot study. Curr Gerontol Geriatr Res 2016;2016:1520932.

18. Dutra MC, Ribeiro RDS, Pinheiro SB. Accuracy and reliability of the Pfeffer Questionnaire for the Brazilian elderly population. Dement Neuropsychol 2015;9:176-83.

19. Silven A, Petrus A, Villalobos-Quesada $\mathrm{M}$, et al. Telemonitoring for patients with COVID-19: recommendations for design and implementation. J Med Internet Res 2020;22:e20953.

20. Padilla-Góngora D, López-Liria R, del Pilar D-LM, et al. Habits of the elderly regarding access to the new information and communication technologies. Procedia Soc Behav Sci 2017;237:1412-7.

21. Sun X, Yan W, Zhou H, et al. Internet use and need for digital health technology among the elderly: a cross-sectional survey in China. BMC Public Health 2020;20:1386.

22. Cornwell EY, Waite LJ. Social network resources and management of hypertension. J Health Soc Behav 2012;53: 215-31.

23. Leeson GW. The comparison in the effect of use of internet and social support on the depression of the urban elderly: evidence from the sample survey of three cities in West China. Special Zone Econ Issue 2018:89-91.

24. Heo J, Chun S, Lee S, et al. Internet use and well-being in older adults. Cyberpsychol Behav Soc Netw 2015;18: 268-72.

25. Kraaijkamp J, van Dam van Isselt E, Persoon A, et al. eHealth in geriatric rehabilitation: systematic review of effectiveness, feasibility, and usability. J Med Internet Res 2021;23:e24015. 